\title{
Clinical and Imaging Analysis of Primary Tumors of the Clavicle
}

\author{
Bao-Hai Yu ${ }^{1}$, Shu-Man Han $^{1}$, Tao Sun ${ }^{1}$, Lei Cao ${ }^{1}$, Hui-Zhao Wu ${ }^{1}$, Yun-Heng Shi ${ }^{1}$, Jin-Xu Wen ${ }^{1}$, Wen-Juan \\ $\mathrm{Wu}^{1, *}$ and Bu-Lang Gao ${ }^{1}$ \\ ${ }^{1}$ Department of Radiology, The Third Hospital of Hebei Medical University, Shijiazhuang, China \\ "Corresponding author: Department of Radiology, The Third Hospital of Hebei Medical University, Shijiazhuang, China. Email: wenjwu@163.com
}

Received 2019 October 12; Revised 2020 May 04; Accepted 2020 May 16.

\begin{abstract}
Background: The clinical and imaging presentations of primary tumors and tumor-like lesions in the clavicle are not clear. Objectives: To investigate the clinical and imaging presentations of primary tumors and tumor-like lesions in the clavicle for better diagnosis.

Patients and Methods: Patients with clavicular diseases between 1963 and 2018 confirmed by pathology who had plain radiography, computed tomography (CT) scan and magnetic resonance imaging were enrolled. The age, sex, imaging data, radiological signs and location of the tumor at the clavicle of patients were analyzed and compared.

Results: Eighty-one patients with clavicular diseases were identified including 49 males and 32 females with an age range of 2 - 76 years (mean, 42.6). There were malignant tumors in 50 cases (61.7\%), intermediate in 12 (14.8\%), and benign in 19 cases (23.5\%). Malignant tumors included metastatic tumors in 31 cases (38.3\%), plasma-cell myeloma in ten (12.3\%), and osteosarcoma in four (4.9\%). The intermediate bone tumors were mainly eosinophilic granuloma in six cases (7.4\%), and aneurysmal bone cyst in three (3.7\%). Benign tumors were mainly osteochondroma (6.2\%), bone cyst (3.7\%), and chondroma (3.7\%). Malignant tumors had bone destruction, periosteal reaction, ill-defined margins and soft tissue masses. Benign tumors and tumor-like lesions had well-defined margins. Conclusion: Many tumors may occur at the clavicle with more malignant than benign tumors with no specific imaging features in most lesions. A lesion near the sternal end with atypical benign imaging features should be considered as malignant.
\end{abstract}

Keywords: Tumors, Tumor-Like Lesion, Clavicle, Imaging, Malignant, Benign

\section{Background}

Primary tumors and tumor-like lesions are rare in the clavicle which is an unusual bone with development and structures different from other tubular bones in many aspects (1). This bone is the first to ossify in the embryo probably at five months. In the first five years of life, further development happens in the diaphysis from intramembranous ossification, and later, typical endochondral ossification of tubular bone occurs at the sternal and acromial ends. Radiologically, evident epiphyses only appear at the sternal end while no epiphysis center appears at the acromial end. The clavicle interior is occupied by coarse cancellous bone with actually no cavity (2). The clavicle has sparse vascular supply with minimal red marrow surrounded by thick cortices of compact bone. These unusual features in the development and structures may account for the uncommon occurrence of tumors and tumor-like lesions in the clavicle $(1,2)$. Bone lesions in the clavicle are rare and not common even though every kind of bone lesion which occurs in the skeleton bone has been described in the clav- icle. Many clavicular lesions have been reported in the literature despite that most articles are of single case reports or very small number of patients (3-15) even though some larger samples have been reported $(1,2,16)$. The great variety of diseases in the clavicle makes radiological diagnosis challenging especially because of limited experience of individual radiologists with these tumors and tumor-like lesions.

\section{Objectives}

This study investigated patients with tumors and lesions resembling tumors in the clavicle over a period of forty years and analyzed the clinical and imaging characteristics of these diseases in order to increase the diagnostic accuracy.

\section{Patients and Methods}

This retrospective study was approved by the ethics committee of our hospital with all the patients given their 
signed informed consent. Between January 1963 and December 2018, patients with tumors in the clavicle confirmed by pathology were enrolled. Inclusion criteria were patients with clavicle tumors who had radiological examinations including plain radiography, computed tomography (CT) scan and magnetic resonance imaging (MRI). Exclusion criteria were infectious diseases, non-infectious inflammatory diseases, trauma and fracture of the clavicle. Age, sex, radiological signs and location of the tumor at the clavicle of the patients were analyzed and compared. For CT scanning, the Siemens 64-detector scanner (Siemens, Germany) was used with the scanning parameters of slice thickness $5 \mathrm{~mm}$, slice interval $5 \mathrm{~mm}$, tube voltage $120 \mathrm{kV}$, tube current $260 \mathrm{~mA}$, window width 2000 and window level 500. For MRI scanning, the Siemens symphony $1.5 \mathrm{~T}$ scanner was used with the following parameters: T1 weighted image (T1WI): repetition time (TR)/echo time (TE) $690 \mathrm{~ms} / 12 \mathrm{~ms}$, T2WI: TR/TE $5000 \mathrm{~ms} / 60 \mathrm{~ms}$, slice thickness $5 \mathrm{~mm}$, slice interval $5 \mathrm{~mm}$, field of view $24 \mathrm{~cm} \times$ $24 \mathrm{~cm}$, and matrix $256 \times 256$.

\subsection{Statistical Analysis}

Statistical analysis was performed with the SPSS ver. 24.0 (IBM, Chicago, IL, USA). Enumeration data were presented as number and percentages and tested with Fisher exact probability method, chi-square for paired $t$-test for significant difference between benign, malignant and intermediate tumors and other data. The significant $\mathrm{P}$ was set as $<0.05$.

\section{Results}

A total of 81 patients with tumors in the clavicle were identified including 49 males and 32 females with an age range of 2 - 76 years of age (mean $=42.6$ ) and a male-tofemale ratio of 1.53:1. There were malignant tumors in 50 cases (61.7\%), intermediate tumors in 12 (14.8\%) and benign tumors in 19 cases (23.5\%) (Figures 1-3), with one peak age period of 11 - 20 years in 21 cases (25.9\%) and another age peak period of 51 - 60 years in 37 cases (45.7\%). Malignant tumors included metastatic tumors (38.3\%), plasma-cell myeloma (12.3\%), and osteosarcoma (4.9\%). The intermediate bone tumors were mainly eosinophilic granuloma (7.4\%) and aneurysmal bone cyst (3.7\%), and according to the World Health Organization (WHO) classification of tumors of soft tissue and bone, intermediate bone tumors indicate those which are locally aggressive but rarely metastasizing (17). Benign tumors were mainly osteochondroma (6.2\%), bone cyst (3.7\%) and chondroma (3.7\%) (Table 1).
The mean age was 53.5 years for 50 cases with malignant tumors, 31.4 years for twelve cases with intermediate tumors and 23.7 years for 19 cases with benign tumors. The mean age was 56.5 years for metastatic tumors, 57.3 for plasma-cell myeloma, 39.3 for osteosarcoma, 17.5 for Ewing's sarcoma, 15.6 for eosinophilic granuloma, and 19.5 for osteochondroma.

The clavicle was divided into three segments as sternal end, middle segment and acromial end with each segment accounting for $1 / 3$ of the clavicle. The location of the lesion was determined according to the position of the lesion center. More malignant tumors occurred in the sternal end than either the intermediate malignant $(\mathrm{P}=0.013)$ or the benign tumors $(\mathrm{P}=0.011)$ (Table 2$)$. Fifty $(61.7 \%)$ lesions were located at the sternal end, with 38 malignant tumors (76\%) including metastatic tumors in 26 cases, plasma-cell myeloma in six, osteosarcoma in three, lymphoma in two and Ewing's sarcoma in one. Among 81 cases, plain radiography was performed in 39 cases, of whom CT scan was conducted in 31, and MRI scan in 20 (Table 3). The bone destruction on CT imaging was in line with what was found on plain radiography, and the lesion had equal to low signal on T1WI but high signal on T2WI on MRI.

Among 22 cases with metastatic tumors (Figure 1), osteolytic destruction occurred in fifteen cases, and mixed destruction in six cases. Expansile destruction took place in fourteen patients, with pathological fracture in four cases. A soft tissue mass occurred in thirteen cases, with apparent sclerosis and periosteal reaction in one patient. In five patients with plasma cell myeloma (Figure 2), the lesion was osteolytic with expansile destruction, ill-defined margins and thinned cortex in three cases. Pathological fracture occurred in three cases with accompanied soft tissue masses. Among four cases with eosinophilic granuloma (Figure 2), three lesions had well-defined margins, but one lesion had ill-defined margins. In three children, the eosinophilic granuloma lesion was expansile with apparent periosteal reaction and bone shell formation. In the remaining two adult patients, the lesion was limited with slight periosteal reaction and no masses in the soft tissue. Malignant tumors had a significantly $(\mathrm{P}=0.003)$ lower incidence in periosteal reaction but a significantly higher incidence in soft tissue mass than intermediate malignant tumors (Table 4).

In two cases with osteosarcoma (Figure 3), one case had osteolytic destruction with a soft tissue mass and tumorous bone, and the other case had mixed bone destruction with ill-defined margins, a soft tissue mass and tumorous bone. In the case of Ewing's sarcoma (Figure 3), the lesion was osteolytic expansile destruction with ill-defined 

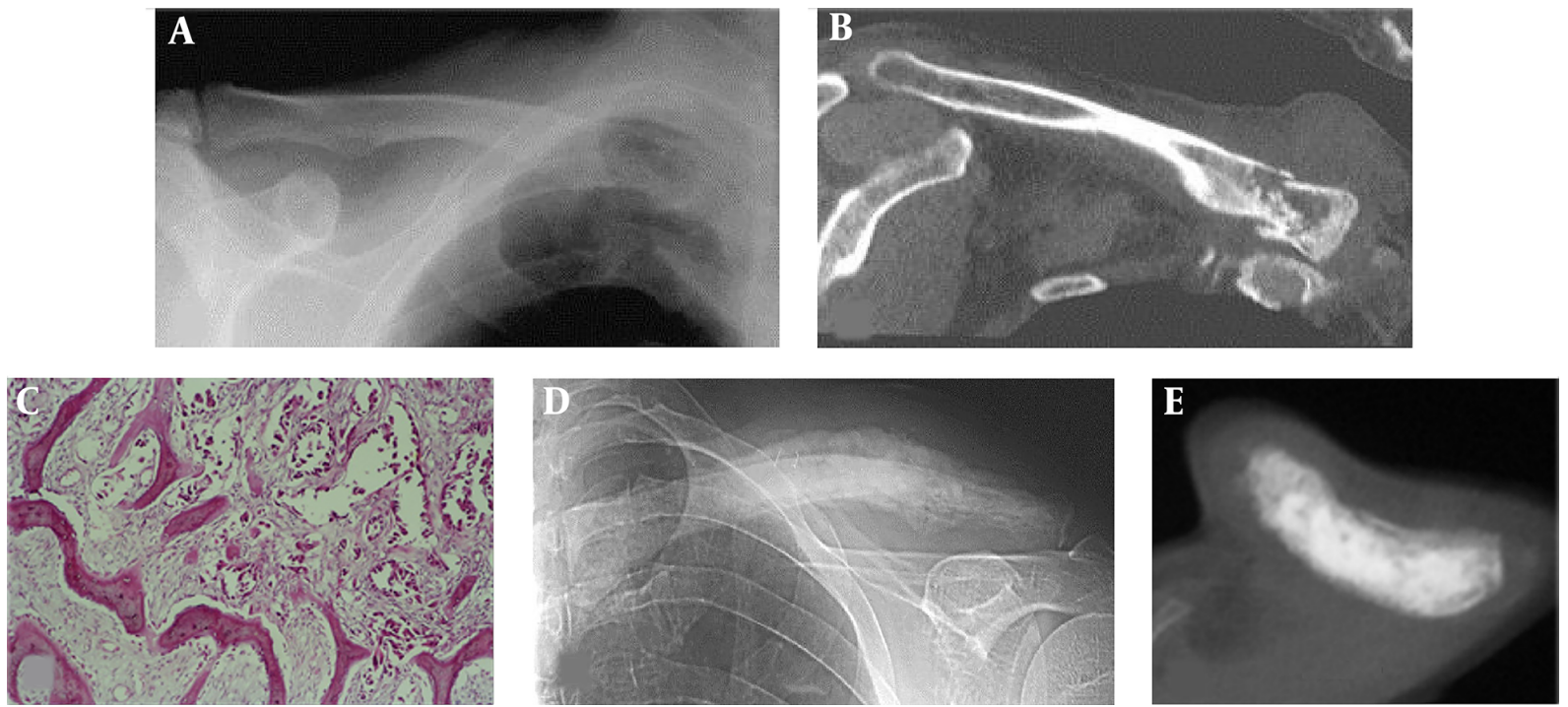

Figure 1. Metastatic tumors. A and B, At the sternal end of the right clavicle, osteolytic bone destruction is revealed with ill-defined margins, pathologic fracture and a soft tissue mass; C, Microscopy of the clavicle in (A) and (B) reveals metastatic adenocarcinoma of the lung with cancer cells forming an adenoid structure surrounded by reactive new bone; D and E, At the middle segment of the left clavicle, osteosclerotic bone destruction is shown with ill-defined margins, apparent periosteal reaction but no soft tissue masses.
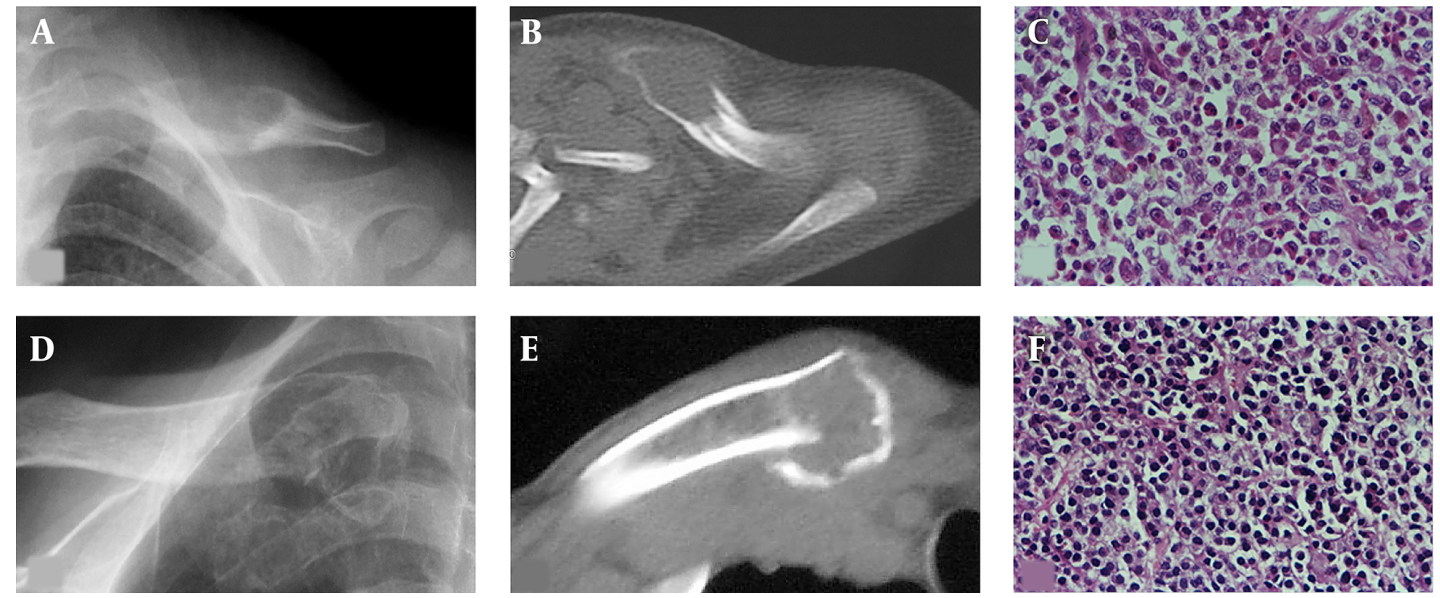

Figure 2. A-C, Eosinophilic granuloma. D-F, plasma-cell myeloma; A-C, An eosinophilic granuloma lesion is demonstrated at the middle segment of the left clavicle with osteolytic bone destruction, ill-defined margins, apparent periosteal reaction but no soft tissue masses (A and B); C, Microscopy of the eosinophilic granuloma shows many Langerhans cells of medium size with eosinophilic cytoplasm and round or oval nuclei which had nuclear groove. Scattered or clustered eosinophils are seen in the background; D-F, A plasma-cell myeloma is shown near the sternal end of the right clavicle with osteolytic, expansile bone destruction, ill-defined margins and pathologic fracture (D and E); F, Microscopically, the lesions of the plasma-cell myeloma are composed of tumor plasmocytes with deviated nuclei.

margins, thinned and discontinued cortex but no soft tissue masses. The aneurysmal bone cyst had eccentric, expansile lytic bone destruction with well-defined margins but no soft tissue masses. The simple bone cyst was expansile lytic bone destruction with well-defined margins. One osteochondroma presented as a bony protrusion like a cauliflower at the distal clavicular end. One case with chondroma at the sternal end had expansile bone destruction with no calcification nor soft tissue swelling.

\section{Discussion}

\subsection{Lesion Nature}

In this study, the most frequent lesion is malignant tumors in 50 cases (61.7\%) including primarily metastatic tumor, plasma-cell tumor and osteosarcoma accounting for 

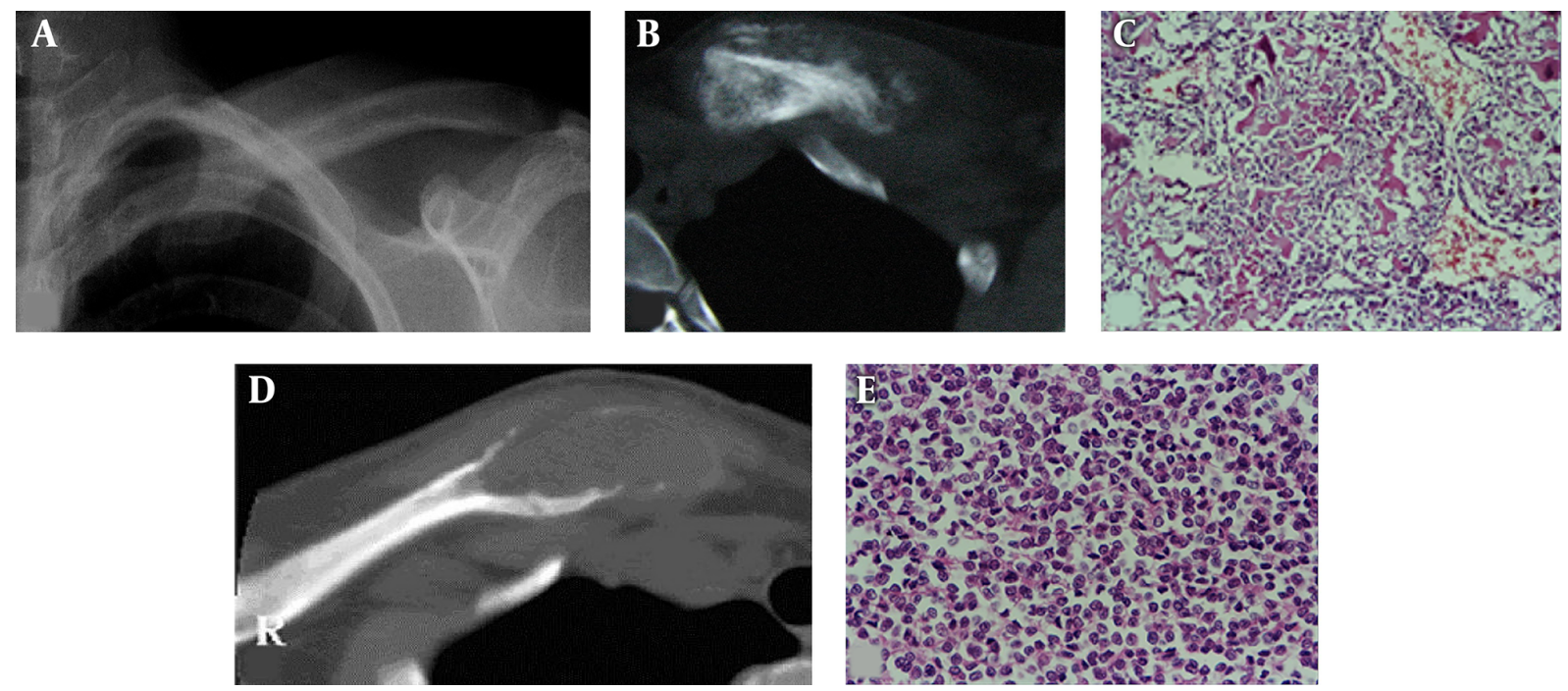

Figure 3. A-C, Osteosarcoma; D and E, Ewing's sarcoma; A and B, An osteolytic destruction is demonstrated at the sternal end of the left clavicle with ill-defined margins, periosteal reaction, a lot of tumorous bone and soft tissue masses; C, Microscopically, the osteosarcoma is composed of tumor cells and tumor-like bone tissue which is produced directly by the tumor cells; D, An osteolytic, expansile destruction lesion is shown at the sternal end of the right clavicle with ill-defined margins, but no periosteal reaction and soft tissue masses; E, Microscopically, the Ewing's sarcoma is composed of small round cells of the same size and shape, with few cytoplasm and fine nuclear chromatin.

$38.3 \%, 12.3 \%$ and $4.9 \%$, respectively. Fibrous dysplasia and giant cell tumor which are common in other body parts are rare in the clavicle accounting for only $1.2 \%$ and $2.5 \%$, respectively, which is probably related to the flat bone of the clavicle. Based on the unpublished data in our hospital over 45 years, the tubular long bones are predominated by benign lesions while most flat and irregular bones like ribs, ilium and sacrum mainly have malignant tumors. The incidence of metastatic tumors and myeloma in the clavicle is similar to that in the ribs, probably in relation to the tissue and anatomical features of the clavicle which is actually a flat bone. All kinds of lesions which tend to occur in flat bones may take place in the clavicle, whereas fibrous dysplasia, giant cell tumors and chondroblastoma which tend to occur in long tubular bones are rare in the clavicle (1-4, 6, 7, 9-12, 14-16). In a study investigating the histopathology, anatomy and epidemiology of bone tumors of the clavicle in 113 patients with a mean age of 40 years (18), 22 benign, 31 intermediate and 60 malignant tumors were found, with eosinophilic granuloma as the most common neoplasm (18.6\%), followed by bone metastases (15.0\%), plasma cell myeloma (8.8\%), Ewing's sarcoma (8.8\%) and osteosarcoma (8.0\%). In this study, 53\% of the tumors were malignant, with a mean age of 51 years in patients with malignant tumors and 28 years in patients with a benign/ intermediate lesion $(\mathrm{P}<0.001)$.

\subsection{Location of Lesions}

Distribution of lesions in the clavicle also has specific characteristics. In our study, 50 (61.7\%) lesions involved the sternal end of the clavicle, including 38 malignant tumors (76\%), which indicates that most of the clavicular lesions occur in the sternal end especially malignant tumors, consistent with the study by Smith et al. (1) This is probably related to the rapid growth and appearance of the epiphysis center only at the sternal end. Because of sparse blood supply to the clavicle (2), metastatic tumors at this site are rare, but in our study, metastatic tumors in the clavicle had the greatest incidence of $38.3 \%$. This is in comparison with other rare diseases in the clavicle because metastatic tumors have a high incidence. Clavicular tumors may easily cause pathological fractures. Traumatic fracture of the clavicle usually occurs in the middle and external segments, and clavicular fracture in the internal segment near the sternal end should raise suspicion of pathological fractures caused by metastatic tumors and plasmacell myeloma, especially in middle and old citizens. In another study (18), the lateral third of the clavicle was most frequently involved.

\subsection{Imaging Presentations}

In the anteroposterior radiograph, the external segment of the clavicle does not overlap with other bones and could be displayed clearly; however, the internal segment 


\begin{tabular}{|c|c|c|c|c|c|c|}
\hline Disease & Percentage & M:F & Age range (mean) & Proximal Third & Middle Third & Distal Third \\
\hline \multicolumn{7}{|l|}{ Malignant tumor } \\
\hline Metastatic $(n=31)$ & 38.3 & 19:12 & $4-76(56.5)$ & 26 & 3 & 2 \\
\hline $\operatorname{PCM}(\mathrm{n}=10)$ & 12.3 & $8: 2$ & $29-67(57.3)$ & 6 & 1 & 3 \\
\hline Osteosarcoma $(n=4)$ & 4.9 & $2: 2$ & $15-60(39.3)$ & 3 & & 1 \\
\hline Lymphoma $(\mathrm{n}=2)$ & 2.5 & $1: 1$ & $34-72(53)$ & 2 & & \\
\hline Ewing's sarcoma $(\mathrm{n}=2)$ & 2.5 & 2:0 & $17-18(17.5)$ & 1 & & 1 \\
\hline Chondrosarcoma $(\mathrm{n}=1)$ & 1.2 & 1:0 & $52(52)$ & & & 1 \\
\hline \multicolumn{7}{|l|}{ Intermediate tumor } \\
\hline Eosinophilic granuloma $(n=6)$ & 7.4 & $3: 3$ & $2-32(15.6)$ & 3 & 2 & 1 \\
\hline $\mathrm{ABC}(\mathrm{n}=3)$ & 3.7 & $1: 2$ & $15-45(34.3)$ & 1 & & 2 \\
\hline Giant cell tumor $(n=2)$ & 2.5 & $2: 0$ & $25-60(42.5)$ & & & 2 \\
\hline Chondromyxoid fibroma $(\mathrm{n}=1)$ & 1.2 & $0: 1$ & $45(45)$ & & 1 & \\
\hline \multicolumn{7}{|l|}{ Benign tumor } \\
\hline Osteochondroma $(\mathrm{n}=5)$ & 6.2 & $2: 3$ & $11-33(19.5)$ & 4 & & 1 \\
\hline Bone cyst $(\mathrm{n}=3)$ & 3.7 & $1: 2$ & $11-15(12.7)$ & & 1 & 2 \\
\hline Chondroma $(\mathrm{n}=3)$ & 3.7 & $2: 1$ & $12-36(21.7)$ & 1 & 1 & 1 \\
\hline Ossifying fibroma $(\mathrm{n}=2)$ & 2.5 & $1: 1$ & $13-16(14.5)$ & 1 & & 1 \\
\hline Osteoid osteoma $(\mathrm{n}=1)$ & 1.2 & 1:0 & $18(18)$ & & 1 & \\
\hline Hemangioma $(n=1)$ & 1.2 & 1:0 & $50(50)$ & & 1 & \\
\hline Fibrous histiocytoma $(\mathrm{n}=1)$ & 1.2 & $0: 1$ & $60(60)$ & 1 & & \\
\hline Fibrous dysplasia $(\mathrm{n}=1)$ & 1.2 & 1:0 & $13(13)$ & 1 & & \\
\hline Lipoma $(\mathrm{n}=1)$ & 1.2 & $0: 1$ & $58(58)$ & & & 1 \\
\hline Osteoma $(\mathrm{n}=1)$ & 1.2 & $1: 0$ & $22(22)$ & & & 1 \\
\hline Total & 100 & 49:32 & $2-76(42.6)$ & 50 & 11 & 20 \\
\hline
\end{tabular}

Abbreviations: ABC, aneurysmal bone cyst; F, female; M, male; PCM, plasma-cell myeloma.

\begin{tabular}{|c|c|c|c|c|c|c|c|}
\hline Tumor & Male & Female & Left & Right & Proximal Third & Middle Third & Distal Third \\
\hline Malignant tumor $(n=50)$ & $66.0 \%(33 / 50)$ & $34.0 \%(17 / 50)$ & $56.0 \%(28 / 50)$ & $44.0 \%(22 / 50)$ & $76 \%(38 / 50)$ & $8.0 \%(4 / 50)$ & $16 \%(8 / 50)$ \\
\hline Intermediate tumor $(\mathrm{n}=\mathbf{1 2})$ & $50.0 \%(6 / 12)$ & $50.0 \%(6 / 12)$ & $58.33 \%(7 / 12)$ & $41.67 \%(5 / 12)$ & $33.3 \%(4 / 12)$ & $25.0 \%(3 / 12)$ & $41.7 \%(5 / 12)$ \\
\hline Benign tumor $(n=19)$ & $52.63 \%(10 / 19)$ & $47.37 \%(9 / 19)$ & $42.11 \%(8 / 19)$ & $57.89 \%(11 / 19)$ & $42.1 \%(8 / 19)$ & $21.05 \%(4 / 19)$ & $36.8 \%(7 / 19)$ \\
\hline
\end{tabular}

${ }^{\mathrm{a}}$ Fisher exact probability test was used. More malignant tumors occurred in the inner area than either the intermediate malignant $(\mathrm{P}=0.013)$ or the benign tumors $(\mathrm{P}=$ 0.011). No other significant $(\mathrm{P}>0.05)$ difference existed between any other two features.

does overlap with ribs and cannot be clearly displayed even in the oblique radiograph. CT scan is needed for further evaluation of the clavicle.

\subsection{Metastatic Tumors}

In our study, the metastatic tumors occurred mostly in patients over 50 years of age. The age was older compared to other bone metastases. These tumors mostly had expansile osteolytic destruction with discontinuation of the cortex and soft tissue masses (8). CT displayed lytic bone destruction with ill-defined margins, a soft tissue mass, discontinued cortex and a primary tumor. In some cases with osteosclerotic metastasis, the lesion had apparent osteosclerosis, bone destruction and a lot of periosteal reac- 


\begin{tabular}{|c|c|c|c|c|c|c|c|c|}
\hline & Lytic destruction & Expansile destruction & Sclerotic destruction & Mixed destruction & IDR & PR & PF & STM \\
\hline Metastatic $(n=22)$ & 15 & 14 & 1 & 6 & 17 & 2 & 4 & 15 \\
\hline $\operatorname{PCM}(\mathbf{n}=5)$ & 5 & 4 & - & - & 5 & - & 3 & 3 \\
\hline Osteosarcoma $(n=2)$ & 1 & 1 & - & 1 & 2 & - & - & 2 \\
\hline Ewing's sarcoma $(n=1)$ & 1 & 1 & - & - & 1 & - & - & - \\
\hline$E G(n=4)$ & 4 & 4 & - & - & 2 & 4 & - & - \\
\hline $\operatorname{ABC}(n=2)$ & 2 & 2 & - & - & - & - & - & - \\
\hline Bone cyst $(n=1)$ & 1 & 1 & - & - & 1 & - & - & - \\
\hline Osteochondroma $(n=1)$ & - & - & - & - & - & - & - & - \\
\hline Chondroma $(n=1)$ & 1 & 1 & 1 & & & & & \\
\hline
\end{tabular}

Abbreviations: ABC, aneurysmal bone cyst; EG, eosinophilic granuloma; IDR, ill-defined rim; PCM, plasma-cell myeloma; PF, pathologic fracture; PR, periosteal reaction; STM, soft tissue mass.

\begin{tabular}{|c|c|c|c|c|c|c|c|c|}
\hline Tumor & Lytic & Sclerotic & Mixed & Expansile & IDR & PR & PF & STM \\
\hline Malignant tumor $(n=30)$ & $73.33 \%(22 / 30)$ & $3.33 \%(1 / 30)$ & $23.33 \%(7 / 30)$ & $66.67 \%(20 / 30)$ & $83.33 \%(25 / 30)$ & $6.67 \%(2 / 30)$ & $23.33 \%(7 / 30)$ & $66.67 \%(20 / 30)$ \\
\hline $\begin{array}{l}\text { Intermediate tumor }(n= \\
\text { 6) }\end{array}$ & $100 \%(6 / 6)$ & 0 & 0 & $100 \%(6 / 6)$ & $33.33 \%(2 / 6)$ & $66.67 \%(4 / 6)$ & 0 & 0 \\
\hline Benign tumor $(n=3)$ & $66.67 \%(2 / 3)$ & $33.33 \%(1 / 3)$ & 0 & $66.67 \%(2 / 3)$ & $33.33 \%(1 / 3)$ & 0 & 0 & 0 \\
\hline Pvalue & 0.391 & 0.170 & 0.463 & 0.193 & 0.013 & 0.005 & 0.463 & 0.000 \\
\hline
\end{tabular}

Abbreviations: IDR, ill-defined rim; PF, pathologic fracture; PR, periosteal reaction; STM, soft tissue mass.

${ }^{a}$ Fisher exact probability test was used. Malignant tumors had a significantly $(P=0.003)$ lower incidence in PR but a significantly higher incidence in STM than intermediate malignant tumors. No other significant $(\mathrm{P}>0.05)$ difference existed between any other two features.

tion, similar to osteosarcoma (Figure 1).

\subsection{Eosinophilic Granuloma}

Eosinophilic granuloma is not frequently found in the clavicle; however, it has been described in detail in the literature $(4,7,12,15)$. The lesion most commonly occurs in children less than 10 years, but it also occurs in much older patients (1). The lesion has well defined margins, homogeneous density inside the lesion, apparent periosteal reaction in children and sclerotic rims. The lesion is usually adjacent to normal bone tissue with no soft tissue masses (Figure 2). Combination of the age of onset and imaging features can readily lead to the correct diagnosis in children rather than in adults because of no imaging specificity.

\subsection{Plasma-Cell Myeloma}

In our study, these patients were mostly more than 50 years and the lesion was lytic expansile bone destruction with ill-defined margins, thinned cortex and concurrent pathological fracture at the sternal end (Figure 2). It is difficult to differentiate it from metastatic tumors. Medullary biopsy and IgM detection in blood or urine may be helpful for diagnosis.

\subsection{Osteosarcoma}

In our series, patients with osteosarcoma over 20 years of age accounted for $71.4 \%$; while, those over 30 years of age accounted for $57.1 \%$ of all osteosarcoma patients, with the age of prevalence greater than for patients with osteosarcoma in long bones (19). Imaging presentations of osteosarcoma are osteolytic destruction with tumorous bone formation and periosteal reaction (Figure 3). Mixed bone destruction can also be shown without periosteal reaction or tumorous bone. A soft tissue mass may be present, similar to the imaging presentations of metastatic tumors with difficulty in differentiation. Clavicular metastatic tumors may have imaging presentations similar to those of osteosarcoma (Figure 1).

\subsection{Ewing's Sarcoma}

The prevalence of Ewing's sarcoma in our study was between 10 and 20 years, consistent with the age of onset for this sarcoma $(20,21)$. The imaging presentations in one such patient was expansile bone destruction with thinned 
discontinued cortex, ill-defined margins but no soft tissue masses (Figure 3). These imaging features are not specific for Ewing's sarcoma and may present difficulty in diagnosis.

In conclusion, many tumors and tumor-like lesions may occur at the clavicle with more malignant than benign tumors with no specific imaging features in most lesions, and a lesion near the sternal end with atypical benign imaging features should be considered as malignant.

\section{Footnotes}

Authors' Contributions: Study design: Bao-Hai Yu, WenJuan Wu, and Bu-Lang Gao. Data collection and analysis: Bao-Hai Yu, Shu-Man Han, Tao Sun, Lei Cao, and Wen-Juan Wu. Study supervision: Hui-Zhao Wu, Yun-Heng Shi, and Jin-Xu Wen. Writing of the paper: Bao-Hai Yu. Revision: BuLang Gao. Approval of the paper: All authors.

Conflict of Interests: The authors declared that there is no conflict of interests in publication of this article.

Ethical Approval: This study was approved by the Ethics Committee of our hospital with all the patients given their signed informed consent. The code is 20180025.

Funding/Support: Nothing to declared.

Informed Consent: Informed consent was obtained from all participants.

\section{References}

1. Smith J, Yuppa F, Watson RC. Primary tumors and tumor-like lesions of the clavicle. Skeletal Radiol.1988;17(4):235-46. [PubMed:3062792].

2. Ogden JA, Beall JK, Conlogue GJ, Light TR. Radiology of postnatal skeletal development. IV. Distal radius and ulna. Skeletal Radiol. 1981;6(4):255-66. [PubMed: 7292022]

3. Akinsulire AT, Badmus OO, Giwa SO. Giant cell tumour of the clavicle: A rare presentation of a locally aggressive tumour. Niger Postgrad Med J. 2017;24(4):250-3. doi: 10.4103/npmj.npmj_133_17. [PubMed: 29355166].

4. Aprin H, Calandra J, Mir R, Lee JY. Radiation-induced chondrosarcoma of the clavicle complicating Hodgkin's disease. A case report. Clin Orthop Relat Res.1986;(209):189-93. [PubMed: 3731593].

5. Bajpai J, Saini S, Bajpai A, Khera R. Rare presentation of giant cell tumor of bone in the lateral end of the clavicle. Am J Case Rep. 2013;14:235-7. doi: 10.12659/AJCR.889121. [PubMed: 23875059]. [PubMed Central: PMC3715393].

6. Eyesan SU, Katchy AU, Idowu OO, Adesina SA, Ayandele BO, AnoEdward U. Non-ossifying fibroma of the right clavicle. Niger Postgrad Med J.2018;25(2):126-9.doi:10.4103/npmj.npmj_60_18.[PubMed: 30027926].
7. Faure C, Beaufils F. [Osseous manifestations of histiocytosis X (radiological signs)]. Poumon Coeur. 1969;25(7):805-35. French. [PubMed: 5401359].

8. Glover TE, Butel R, Bhuller CM, Senior EL. An unusual presentation of adenoid cystic carcinoma of the breast with metastatic disease in the clavicle. BJR Case Rep. 2017;3(2):20160119. doi: 10.1259/bjrcr.20160119. [PubMed: 30363306]. [PubMed Central: PMC6159263].

9. Kaiser CL, Yeung CM, Raskin KA, Lozano-Calderon SA. Aneurysmal bone cyst of the clavicle: A series of 13 cases. J Shoulder Elbow Surg. 2019;28(1):71-6. doi:10.1016/j.jse.2018.06.036. [PubMed: 30243904].

10. Kumar A, Arora N, Pandove PK, Anand G, Arora B. Pulsatile Clavicular Swelling as the Sole Presentation of Asymptomatic Renal Cell Carcinoma with an Isolated Metastasis to the Clavicle: A Case Report. J Clin Diagn Res. 2016;10(12):PD07-8. doi: 10.7860/JCDR/2016/23220.9064. [PubMed: 28208928]. [PubMed Central: PMC5296501].

11. Moreira AC, Suster DI, Eide SE, Rosenthal DI, Chang CY. Osteoblastoma of the clavicle at the site of a previous fracture-first case report and review of the literature. Skeletal Radiol. 2019. doi: 10.1007/s00256-01903197-x. [PubMed: 30850870].

12. Prager PJ, Menges V, DiBiase M, Wurster KH, Krastel A, Assmus H. [Eosinophilic granuloma of the bone in adults (author's transl)]. Radiologe.1976;16(1):21-8. German. [PubMed:1257426].

13. Recine F, Bongiovanni A, Casadei R, Pieri F, Riva N, De Vita A, et al. Primary leiomyosarcoma of the bone: a case report and a review of the literature. Medicine (Baltimore). 2017;96(45). e8545. doi: 10.1097/MD.0000000000008545. [PubMed: 29137065]. [PubMed Central: PMC5690758].

14. Sadacharan D, Mahadevan S, Kabeer KK, Sundarraman G. A case of pulsating sternal and clavicular metastases of thyrofollicular carcinoma. BMJ Case Rep. 2017;2017. doi: 10.1136/bcr-2017-220010. [PubMed: 28385736].

15. Sugi MT, Fedenko AN, Menendez LR, Allison DC. Clavicular eosinophilic granuloma causing adult shoulder pain. Rare Tumors. 2013;5(1). e8. doi: 10.4081/rt.2013.e8. [PubMed: 23772307]. [PubMed Central: PMC3682460].

16. Terra BB, Rodrigues LM, Padua DV, Sassine TJ, Cavatte JM, De Nadai A. Osteoid osteoma of the distal clavicle. Rev Bras Ortop. 2017;52(2):2104. doi: 10.1016/j.rboe.2017.01.006. [PubMed: 28409140]. [PubMed Central: PMC5380785].

17. World Health Organization. WHO classification of tumours of soft tissue and bone. IARC Press; 2013.

18. Priemel MH, Stiel N, Zustin J, Luebke AM, Schlickewei C, Spiro AS. Bone tumours of the clavicle: Histopathological, anatomical and epidemiological analysis of 113 cases. J Bone Oncol. 2019;16:100229. doi: 10.1016/j.jbo.2019.100229. [PubMed:30976505]. [PubMed Central: PMC6439286]

19. Tan JZ, Schlicht SM, Powell GJ, Thomas D, Slavin JL, Smith PJ, et al. Multidisciplinary approach to diagnosis and management of osteosarcoma - a review of the St Vincent's Hospital experience. Int Semin Surg Oncol. 2006;3:38. doi: 10.1186/1477-7800-3-38. [PubMed: 17081310]. [PubMed Central: PMC1636057].

20. Mittal A, Mehta V, Bagga P, Pawar I. Sunray appearance on sonography in Ewing sarcoma of the clavicle. J Ultrasound Med. 2010;29(3):493-5. [PubMed: 20194947].

21. Weber KL, Makimato A, Raymond AK, Pearson MG, Jaffe N. Ewing sarcoma of the clavicle in a 10-month-old patient. Med Pediatr Oncol. 2000;34(6):445-7. [PubMed:10842256]. 